\title{
Shock Acceleration of Cosmic Rays - a critical review
}

\author{
J.G. Kirk† and R.O. Dendy $\ddagger$ \\ E-mail: John.Kirk@mpi-hd.mpg.de \\ † Max-Planck-Institut für Kernphysik, Postfach 1039 80, D-69029 Heidelberg, \\ Germany \\ $\ddagger$ UKAEA Fusion, Culham Science Centre, Abingdon OX14 3DB, UK
}

\begin{abstract}
Motivated by recent unsuccessful efforts to detect the predicted flux of $\mathrm{TeV}$ gamma-rays from supernova remnants, we present a critical examination of the theory on which these predictions are based. Three crucial problems are identified: injection, maximum achievable particle energy and spectral index. In each case significant new advances in understanding have been achieved, which cast doubt on prevailing paradigms such as Bohm diffusion and single-fluid MHD. This indicates that more realistic analytical models, backed by more sophisticated numerical techniques should be employed to obtain reliable predictions. Preliminary work on incorporating the effects of anomalous transport suggest that the resulting spectrum should be significantly softer than that predicted by conventional theory.
\end{abstract}

\section{Introduction}

Supernova remnants (SNR) are the favourite candidates for the source of 'galactic' cosmic rays (e.g., [1]) and the best way to test this hypothesis is to search for the gamma-ray signal [2]. In the case of hadrons, this is produced by the pions from nuclear collisions; in the case of leptons, by bremsstrahlung or by inverse Compton scattering of the cosmic microwave background radiation. Such a signal should ideally indicate that the nuclear component of cosmic rays in SNR is much younger than that in the interstellar medium, or, failing this, that it is much more intense in SNR than in the interstellar medium. To date, no unambiguous signal has been detected which fulfils either of these criteria.

In order to predict the signal, it is necessary to propose a detailed theory of cosmic ray acceleration, which inevitably involves many simplifying assumptions. Using as a starting point the mechanism of diffusive shock acceleration (for a review see [3]), this task has been undertaken by several groups (e.g., [4, 0, 6, 7, 8, 9, 10, 11]). The predicted flux of $\mathrm{TeV}$ gamma-rays produced by the hadronic component of cosmic rays from a number of nearby SNR lies near the sensitivity limit of present day imaging Cerenkov telescopes. However, the only detections reported to date are of objects where energetic leptons are at least equally plausible as radiating particles [2]. Those objects in which 
hadrons are expected to dominate do not show emission at the level hoped for [12, 4, 13]. In the case of Tycho's supernova remnant the observational upper limit is very close to a recent, conservative prediction of the flux [14, 15].

The indirect arguments in favour of the origin of (hadronic) cosmic rays in SNR (notably the energy budget [16]) remain reasonably convincing. Therefore, in view of the observational situation, it seems appropriate to re-examine the status of the theoretical arguments used to predict the gamma-ray fluxes. In this paper we concentrate on recent progress pertaining to three aspects: the injection problem, the question of the maximum achievable particle energy, and the expected spectral slope of accelerated particles.

\section{The injection problem}

The fundamental assumption of the theory of diffusive shock acceleration is that accelerated particles diffuse in space - i.e., that the particle flux is proportional to the gradient of the particle density (Fick's law). Charged particles deflected by fluctuations in the electromagnetic fields obey this relation only if their velocities are distributed almost isotropically. More precisely, the theory employs an expansion in the ratio of the plasma speed in the shock frame to the particle speed, and the velocity anisotropy is taken to be first order in this small parameter [3]. At a shock front, the downstream plasma speed is of the same order as the thermal speed of the ions in the plasma, so that the theory does not apply to particles whose energy is less than several times the thermal energy. The question of how particles might be accelerated from the thermal pool up to an energy where they can be assumed to diffuse is referred to as the 'injection problem', and cannot be treated within the framework of the diffusive acceleration theory.

Nevertheless, this is an important question both for cosmic ray composition and for the efficiency with which the acceleration process can proceed. In the case of ions, much numerical work has been performed with Monte-Carlo simulations and hybrid codes for a review see [17]. Although it is now generally accepted that the collision operator used by early Monte-Carlo simulations (e.g., 18]) is too simple to describe injection, this technique has the advantage of being tractable in three dimensions. In contrast, hybrid simulations of the shock structure which directly show the ion population emerging from the thermal pool are restricted to two space dimensions. Consequently, cross-field transport is not treated consistently [19], which might be important for the injection mechanism 20].

In addition to numerical work, an analytic theory of injection has been developed by Malkov \& Völk [21]. In this, a thermostat model of the shock is employed in which some fraction of the hot downstream particles is assumed to stream into the upstream plasma. The resulting ion beam excites Alfvén waves via the cyclotron instability, which scatter the particles in pitch-angle. Thus, the physical ingredients are the same as in standard cosmic ray transport theory. However, because of the inherent anisotropy of the mildly suprathermal particles, it is necessary to adopt a more refined treatment of the transport [22]. To complement this model, Malkov [23] has developed a theory of the thermostat 
which involves a large amplitude, monochromatic Alfvén wave, whose role is to confine the majority of hot ions in the downstream region, whilst allowing a small fraction to counterstream into the upstream plasma. This theory has the advantage of offering a prescription suitable for incorporation into combined cosmic ray and hydrodynamic codes 24]. However, the theory contains many simplifications whose validity can be tested only by extensive numerical simulation.

The problem of electron injection has long been considered to be even more difficult than that of ion injection. This is because the cyclotron resonance condition which enables ions to excite weakly damped Alfvén waves cannot be satisfied by mildly suprathermal electrons (see, for example, [21]). Indeed, an electron velocity exceeding roughly 2000 times the Alfvén speed would be required for resonance, which renders the process irrelevant for the problem of injection. A viable alternative has recently been proposed by Dieckmann et al [25], building on suggestions by Galeev [26] and Galeev et al [27] and by McClements et al 28]. Dieckmann et al show that shock-reflected ion populations in the upstream plasma can drive collective instabilities, such that the resultant waves excited in the plasma damp on thermal electrons, thereby accelerating them across the magnetic field to mildly relativistic energies. A fully self-consistent treatment of this fundamentally nonlinear plasma process is obtained using large-scale particle-incell simulations, and there is close quantitative agreement with analytical theory where points of contact exist. The ion population parameters are initialised on the basis of shock acceleration models and parameters, for example those of Cargill \& Papadopoulos [29. Thereafter, broadband electrostatic field oscillations grow in the frequency range between the electron plasma- and gyro-frequencies, excited initially by Buneman-type instabilities, with episodes of high field temporaly correlated with episodes of electron energisation. Several resonant and non-resonant mechanisms for the latter appear to be at work, of which the strongest involves stochastic wave-particle interactions. Dieckman et al demonstrate conclusively that this "bootstrap" mechanism can energise electron populations from background to characteristic energies above $10 \mathrm{keV}$, (sufficient to account for the hard X-ray emission from, for example, Cas-A [30 or from clusters of galaxies [31]) with individual electrons accelerated to several tens of keV. Under typical conditions in the interstellar medium, this would enable them to fulfil the cyclotron resonance condition with weakly damped Alfvén waves.

\section{Maximum achievable energy}

To order of magnitude accuracy, the acceleration timescale $t_{\mathrm{acc}}$ at a shock front can be estimated as

$$
t_{\mathrm{acc}} \approx \kappa / u^{2}
$$

where $\kappa$ is the spatial diffusion coefficient and $u$ is the shock speed. Unfortunately, the value of $\kappa$ is not directly measurable. Furthermore, it depends on the particle energy (through their Larmor radius) and the properties of the plasma turbulence (which is 
probably driven by the accelerated particles themselves [32]), so that it is difficult to estimate reliably. Nevertheless, it is usual to assume that there exists a 'self-quenching' mechanism in operation which limits the amplitude $\delta B$ of the turbulent fluctuations in the magnetic field $B$ near the shock front such that $|\delta B| \lesssim B$ [33, 34]. In this case, one is led to the estimate

$$
\begin{aligned}
\kappa & <\kappa_{\text {Bohm }} \\
& =\frac{2 v^{2} \gamma m c}{15 Z e B}
\end{aligned}
$$

where $v, \gamma, m$ and $Z e$ are the particle's velocity, Lorentz factor, mass and charge and $B$ is the average magnetic field strength.

In the standard picture of cosmic ray acceleration in a supernova remnant, the maximum energy achieved is determined in the free expansion phase (e.g., [35]). If the diffusion coefficient is limited according to Eq. (2), this phase does not really last long enough to allow cosmic rays to be accelerated up to the observed knee in the spectrum, at $\sim 10^{15}-10^{16} \mathrm{eV}$ : setting the acceleration timescale equal to the age of the shock front gives as an estimate of the maximum energy

$$
E_{\max }=6 \times 10^{13} Z\left(\frac{u}{3000 \mathrm{~km} \mathrm{~s}^{-1}}\right)^{2}\left(\frac{t_{\mathrm{sw}}}{300 \mathrm{yr}}\right)\left(\frac{B}{1 \mu \mathrm{G}}\right) \mathrm{eV}
$$

in terms of the shock speed $u$ during the free-expansion phase and the time $t_{\mathrm{sw}}$ available before this phase ends. A more careful analysis - amongst other effects, allowing for the fact that the limit given in Eq. (2) should be reached only at the shock front and not throughout the upstream plasma - has been performed by Lagage \& Cesarsky [35], who conclude that the Eq. (41) is an over estimate by at least a factor of six.

Several papers have suggested ways out of this difficulty, but, until recently, none of these has seemed very promising. Jokipii [36], for example, pointed out that, according to the quasi-linear theory of plasma turbulence, the diffusion coefficients parallel and perpendicular to the magnetic field satisfy

$$
\kappa_{\perp} \ll \kappa_{\text {Bohm }} \ll \kappa_{\|}
$$

so that perpendicular shocks should accelerate particles much faster than parallel ones. However, to make a substantial difference for supernova remnant shocks, this not only would require fine-tuning of the interstellar magnetic field to make the shock front perpendicular, but would also only be effective if, contrary to expectation, the level of turbulence stayed well below the self-quenching limit i.e., if $|\delta B| \ll B$. Using a more conventional approach, Berezhko [37] has presented an re-analysis of the estimate (四), arriving at a value $E_{\max } \approx 10^{15} \mathrm{eV}$. However, much of the enhancement he found arises from the reflecting boundary condition imposed at the so-called 'piston'. This is a numerical device used to drive the exploding remnant in a hydrodynamic simulation, and the boundary condition is chosen for convenience. It is not clear that there is a real physical basis to the effects it produces. Furthermore, Berezhko did not take account of the reduction of $E_{\max }$ by the effects which Lagage \& Cesarsky [35] had found to be important. 
However, in an interesting recent development, Lucek \& Bell [38] have performed numerical simulations of the turbulence driven by cosmic ray streaming at a shock front. This seems to be the only practicable way of advancing our understanding beyond that of weakly nonlinear computations of growth and damping [33, 34]. In particular, it provides an opportunity of testing the 'self-quenching' hypothesis $|\delta B| \lesssim B$ [33], which is the crucial ingredient of the limit given in Eq. (2). The simulations, which are fully 3-dimensional, use an MHD code for the background plasma, together with a kinetic description of the accelerated cosmic rays. Initial conditions are chosen with an idealised energy distribution of isotropic cosmic rays, superposed on a background plasma which streams in the direction of the magnetic field at an Alfvén Mach number of 10. The temporal evolution is followed until the instability saturates. It is found that the linearly most unstable Alfvén wave grows with approximately the linear growth rate until well into the nonlinear regime. The magnetic field of this wave, which is directed perpendicular to the streaming direction, eventually dominates the initial field, causing the instability to saturate when it is strong enough to absorb the energy associated with the streaming. At this stage, $|\delta B| \gg B$, — well above the proposed self-quenching value - and an initially parallel shock front (which is not included explicitly in the simulations) would have become essentially perpendicular.

These results have a major impact on the estimate of $E_{\max }$ described above. The strong amplification of the magnetic field means that in estimating the Bohm diffusion coefficient according to Eq. (3) it is not realistic to insert the value of the magnetic field in the unperturbed interstellar medium. Even if we ignore the difference between a parallel and quasi-perpendicular shock, and set $\kappa_{\|} \sim \kappa_{\perp} \sim \kappa_{\text {Bohm }}$, a substantial increase in $E_{\text {max }}$ results. Lucek \& Bell [38] estimate a field enhancement of a factor of 1000 which, using the estimate of Lagage \& Cesarsky [35] implies $E_{\max } \sim 10^{16} \mathrm{eV}$.

\section{Spectral index}

Adopting an arbitrary power-law distribution for the accelerated particles, Gaisser et al [39] find that the best fit to the EGRET observations of the SNR IC443 and $\gamma$-Cygni is achieved with a cosmic ray spectrum given by $-\mathrm{d} \ln N / \mathrm{d} \ln E \equiv s=2.4$, (where $\mathrm{d} N(E)$ is the differential number of cosmic rays in the remnant with energy between $E$ and $E+\mathrm{d} E$ ) which cuts off at about $80 \mathrm{TeV}$, well above the EGRET energy range. Theoretical models of nonlinear diffusive acceleration, on the other hand, give spectra at the position of the shock front which are rather harder. For example, both stationary Monte-Carlo spectra in plane geometry and time-dependent kinetic computations in spherical geometry agree on the basic shape to be expected [40], which is very hard $(s<2)$ below the cut-off energy. An even harder $(s=1.5)$ spectrum is found in the full analytic solution of the stationary plane case [41, 42] - a difference which might be due to the technical details associated with the assumptions concerning particle escape, but which has not yet been investigated in depth. The gamma-ray emission of a SNR depends not just on the instantaneous spectrum of particles at the shock front, but on a 
superposition of particle spectra throughout the remnant. Nevertheless, the integrated particle spectrum has a slope $s \lesssim 2$, which hardens towards higher energy [6, 11] and could not produce the type of particle spectrum favoured by Gaisser et al [39]. A hard high-energy spectrum ought to be more easily seen at $\mathrm{TeV}$ rather than $\mathrm{GeV}$ energies, but results to date are discouraging [12]. Only two or, perhaps, three shell-type SNR have so far been detected [43, 44, 45] and it is quite possible that this emission arises from relativistic electrons of fairly soft spectrum [46, 47], rather than the predicted hard-spectrum protons.

However, not only the maximum energy, but also the spectral slope of cosmic rays produced by a SNR shock is strongly affected by the properties of the self-induced turbulence if, as suggested in the previous section, the self-quenching mechanism fails to maintain $|\delta B| \lesssim B$. This is because the magnetic field fluctuations generated lie in the plane of the shock front. Acceleration of cosmic rays therefore involves the cross-field transport properties of the plasma. Such a situation has already been investigated in the test-particle approximation [48], where it was found that an anomalous, non-diffusive, transport mode arises due to the statistical wandering or 'braiding' of the magnetic field lines. This had previously been studied in connection with the propagation of cosmic rays through the galactic magnetic field [49, 50, 51] and is well-known in fusion plasmas [52, 53]. Its effect is to soften the spectrum of accelerated particles [54, 55] - in the case of test particles at a shock of compression ratio 4, from the familiar result of diffusive shock acceleration: $s=2$ to the value $s=2.5$. Physically, this arises because the anomalous transport mode inhibits cross-field propagation, and tends to sweep particles away from the shock front into the downstream plasma more effectively than does diffusive transport. No nonlinear calculations of this process have yet been attempted, but a softer spectrum, more in line with that suggested by the gamma-ray observations, is clearly to be expected.

\section{Conclusions}

The standard picture of the diffusive acceleration of cosmic rays at a supernova shock front faces several well-known and stubborn problems. Nevertheless, progress has been substantial, and new perspectives are emerging:

- In the case of electron injection, large scale particle-in-cell simulations have shown that energisation can occur in the turbulence driven by a population of reflected ions 25]. For ion injection, an analytic theory is avaliable which describes the injection process at a parallel shock, given that some fraction of the thermal ions counter-stream [21]. An analytic theory is also under development which aims to provide an understanding of how such counter-streaming ions can be generated self-consistently [23].

- The failure of the standard mechanism to accelerate particles up to $10^{16} \mathrm{eV}$, appears to be due to an underestimate of the importance of self-generated turbulence at the 
Shock Acceleration of Cosmic Rays - a critical review

shock front. Once again, this realisation has emerged from computer simulation work 38.

- The hard spectra predicted in the standard picture, which have not been confirmed by observation, are also based on an assumption about the self-generated turbulence which is now open to question. The modifications which are introduced by anomalous transport properties have been shown to produce softer spectra, more in line with the constraints inferred from observations of gamma-rays [48, 54, 55].

\section{Acknowledgments}

This work is a collaboration of the European Network AstroPlasmaPhysics supported by the European Commission under the TMR-programme, contract number ERBFMRXCT-98-0168, and was supported in part by the UK DTI. We thank Felix Aharonian and Heinz Völk for helpful comments.

\section{References}

[1] Berezinskii V.S., Bulanov S.V., Dogiel V.A., Ginzburg V.L., Ptuskin V.S. 1990 'Astrophysics of Cosmic Rays' (North Holland, Amsterdam)

[2] Aharonian F.A. 1999 Astroparticle Phys. 11, 225

[3] Drury L.O'C. 1983 Rep. Prog. Phys. 46, 973

[4] Drury L.,O'C., Aharonian F.A., Völk H.J. 1994 A\&A 287, 959

[5] Naito T, Takahara F. 1994 J. Phys. G 20, 477

[6] Berezhko E.G., Völk H.J. 1997 Astropart. Phys. 7, 183

[7] Mastichiadis A. 1996 A\&A 305, L53

[8] Pohl M. 1996 A\&A 307, L57

[9] Sturner S.J., Skibo J.G., Dermer C.D., Mattox J.R. 1997 ApJ 490, 619

[10] Berezhko E.G., Völk H.J. 2000 A\&A 357, 283

[11] Ellison D.C., Berezhko E.G., Baring M.G. 2000 ApJ 540, 292

[12] Buckley J.H. et al 1998 A\&A 329, 639

[13] Aharonian F., Drury L.O'C., Völk H.J. 1994 A\&A 285, 645

[14] Rowell G. et al 2000 to appear in Proceedings of the Heidelberg Symposium on Gamma-Ray Astronomy

[15] Völk H.J. 1997 in 'Towards a major atmospheric Čerenkov Detector - V' ed. O.C. de Jager, p. 87 (Potchefstroom University, Potchefstroom)

[16] Axford W.I. 1981 Proc. 17th ICRC (Paris) 12, 155

[17] Scholer M., Kucharek, H., Trattner K.J. 1998 Adv. Space Res. 21, 533

[18] Ellison D.C., Baring M.G., Jones F.C. 1995 ApJ 453, 873

[19] Giacalone J., Jokipii J.R., Kota J. 1994 JGR 99, 19351

[20] Giacalone J., Ellison D.C. 2000 J. Geophys. Res. 105, 12541

[21] Malkov M.A., Völk H.J. 1995 A\&A 300, 605

[22] Kirk J.G., Schneider P. 1989 A\&A 225, 559

[23] Malkov M.A. 1997 Phys. Rev. E 58, 4911

[24] Gieseler U.D.J., Jones T.W., Kang H. 1999 Proc. 26th. ICRC, 4, 419

[25] Dieckmann M.E., McClements K.G., Chapman S.C., Dendy R.O., Drury L.O'C. 2000 A\&A 356, 377

[26] Galeev A.A. 1984 Sov. Phys. J. Exp. Theor. Phys. 59, 965

[27] Galeev A.A., Malkov M.A., Völk H.J. 1995 J. Plasma Phys. 54, 59 
[28] McClements K.G., Dendy R.O., Bingham R., Kirk J.G., Drury L.O'C. 1997 MNRAS 291, 241

[29] Cargill P.J., Papadopoulos K. 1988 ApJ 329, L29

[30] Laming J.M. 2000 astro-ph/0008426, to appear in ApJ

[31] Sarazin C.L., Klempner J.C. 2000 ApJ 533, 73

[32] Bell A.R. 1978 MNRAS 182, 147

[33] Völk H.J. 1981 Proc. 17th ICRC (Paris) OG 4-28

[34] McKenzie J.F., Völk H.J. 1982 A\&A 116, 191

[35] Lagage P.O., Cesarsky C.J. 1983 A\&A 125, 249

[36] Jokipii J.R. 1987 ApJ 313, 842

[37] Berezhko E.G. 1996 Astropart. Phys. 5, 367

[38] Lucek S., Bell A.R. 2000 MNRAS 314, 65

[39] Gaisser T.K., Protheroe R.J., Stanev T. 1998 ApJ 492, 219

[40] Berezhko E.G., Ellison D.C. 1999 ApJ 527, 385

[41] Malkov M.A. 1997 ApJ 485, 638

[42] Malkov M.A., Diamond P.H., Völk H.J. 2000 ApJ 533, L171

[43] Tanimori T. et al 1998 ApJ 497, L25

[44] Puehlhofer G. et al 1999 Proceedings 26th ICRC Eds.: D. Kieda, M. Salamon, B. Dingus (University of Utah, Salt Lake City) 3, 492

[45] Muraishi H. et al 2000 A\&A 354, L57

[46] Aharonian F.A., Atoyan A.M., 1999 A\&A 351, 330

[47] Atoyan A.M., Aharonian F.A., Tuffs R.J., Völk H.J. 2000 A\&A 355, 211

[48] Duffy P., Kirk J.G., Gallant Y.A., Dendy R.O. 1995 A\&A 302, L21

[49] Getmansev G.G. 1963 Sov. Astron. J. 6, 477

[50] Jokipii J.R., Parker E.N. 1969 ApJ 155, 777

[51] Chuvilgin L.G., Ptuskin V.S. 1993 A\&A 279, 278

[52] Isichenko M.B. 1991 Plasma Physics \& Controlled Fusion 33, 795

[53] Rax J.M., White R.B. 1992 Phys. Rev. Letts. 68, 1523

[54] Kirk J.G., Duffy P., Gallant Y.A. 1996 A\&A 314, 1010

[55] Gieseler U.D.J., Kirk J.G. 1999 Proc. 26th. ICRC, 4, 427 